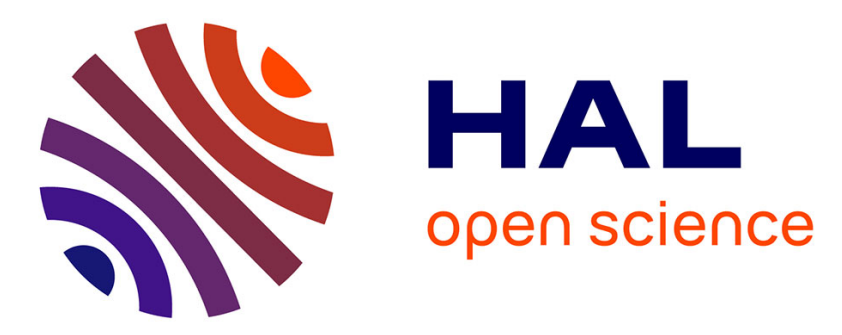

\title{
Capacitive Coupling of Conducting Polymer Tattoo Electrodes with the Skin
}

\author{
Laura M Ferrari, Usein Ismailov, Francesco Greco, Esma Ismailova
}

\section{To cite this version:}

Laura M Ferrari, Usein Ismailov, Francesco Greco, Esma Ismailova. Capacitive Coupling of Conducting Polymer Tattoo Electrodes with the Skin. Advanced Materials Interfaces, 2021, 8 (15), pp.2100352. 10.1002/admi.202100352 . hal-03503743

\section{HAL Id: hal-03503743 \\ https://hal.science/hal-03503743}

Submitted on 28 Dec 2021

HAL is a multi-disciplinary open access archive for the deposit and dissemination of scientific research documents, whether they are published or not. The documents may come from teaching and research institutions in France or abroad, or from public or private research centers.
L'archive ouverte pluridisciplinaire HAL, est destinée au dépôt et à la diffusion de documents scientifiques de niveau recherche, publiés ou non, émanant des établissements d'enseignement et de recherche français ou étrangers, des laboratoires publics ou privés. 


\section{Capacitive coupling of conducting polymer tattoo electrodes with the skin}

Laura M. Ferrari, Usein Ismailov, Francesco Greco and Esma Ismailova*

Dr. L. M. Ferrari

Center for Micro-BioRobotics@SSSA, Istituto Italiano di Tecnologia, 56025, Pontedera, Italy

INRIA, Université Côte d'Azur, 06902, Sophia Antipolis, France

U. Ismailov, Dr. E. Ismailova

Mines Saint-Etienne, Department of Bioelectronics, Centre of Microelectronics in Provence, F-13541, Gardanne, France

E-mail: ismailova@emse.fr

Dr. F. Greco

Institute of Solid State Physics, Graz University of Technology, 8010, Graz, Austria

Center for Micro-BioRobotics@SSSA, Istituto Italiano di Tecnologia, 56025, Pontedera, Italy

Keywords: Conformable biosensors, tattoo electrodes, biosignal transduction, skin impedance, conducting polymers 
Tattoo electronics is one of the emerging technologies in skin compliant biosensing. The growing interest in their large application in health monitoring raises several interrogations on how these sensors interface with the skin. In this paper, we focus on the bioimpedance at the interface of the skin and ultra-conformable tattoo electrodes made of conducting polymers. The electrochemical characteristics of these electrodes differs from traditional gelled $\mathrm{Ag} / \mathrm{AgCl}$ electrodes. The modeling of equivalent circuits in different skin-electrode configurations proposes the explanation of the biopotentials transduction mechanism. The strong agreement between the circuit model and experimental values reveal the capacitive coupling of conducting polymer tattoo electrodes where circuit's values respect the electrodes' and skin physical characteristics. Additional studies underline an enhanced signal stability in inter/intra-subject evaluations using dry tattoos beneficial for broad long-term recordings. This study provides a comprehensive explanation of the skin/tattoo electrode interface model. The understanding of this interface is essential when designing next generation wearable biomonitoring devices using imperceptible interfaces.

\section{Introduction}

Dry or gelled, bulky rigid or conformable electrodes show their thriving application in recording of various surface electrophysiological signals in health monitoring. It expands from the cardiac to the more challenging biopotentials detection coming from the brain. ${ }^{[1-3]}$ The main role of a cutaneous electrode is to sense and accurately translate biosignals inside the body to the electronic acquisition system. Simply, metal plates positioned on moist skin can perform such 
detection since many decades. In such cases, routine abrasive cleaning steps reduce skin impedance and promote signal transduction. On the other hand, the use of ionic hydrogels allows a stable conductive contact between the metal electrode and the skin in practical use. Therefore, gelled $\mathrm{Ag} / \mathrm{AgCl}$ electrodes are considered nowadays as gold medical standards owing to their capability to maintain low impedance contact with the skin and yet high signal quality recordings. ${ }^{[4]}$ The growing need in personalized and minimally invasive medical care pushes the development of novel cutaneous sensors. It derives from the use of dry electrodes with comparable performances as medical $\mathrm{Ag} / \mathrm{AgCl}$ that are better suited for long-term applications. ${ }^{[5]}$ If gelled $\mathrm{Ag} / \mathrm{AgCl}$ electrodes offere to avoid intrusive skin preparation steps in clinical conditions, they lose their performances in long-lasting wearable cutaneous electrophysiology. The gels hydration alters with time, which degrades the signal detection quality while the rigid core of the electrodes makes them sensitive to the motion. A small displacement of the electrode during a movement results in the capacitance value changes because it directly correlates to the distance between the electrode and skin. Flexible electronics offers enabling technologies to design versatile solutions for biomedical applications with compatible mechanical characteristics. ${ }^{[6]}$ Novel ideas appears in designing surface flexible electrodes using metals ${ }^{[2,7]}$ and organic conductors. ${ }^{[8][9]}$ From the fabrication point of view, the metals are usually micropatterned on plastic foils while organic conductors can be directly deposited on low temperature processable substrates using low cost printing techniques. Such processes allow decreasing dimensionalities and proposing thin layer-based electrodes that can achieve intimate contact with the biological tissues. At micrometric scale, these electrodes offer better mechanical and electrical contact than traditional rigid and bulk sensors. ${ }^{[10,11]}$ In tattoo technology, temporary 
tattoo paper is a thin transferable substrate that carries electrical capabilities. ${ }^{[12,13]}$ It creates an unnoticeable and long-lasting interface with the skin, while ensuring an easy yet reliable handling. ${ }^{[1,14]}$ Among the different variations, electronic tattoos are generally composed of multiple materials consecutively layered to create a functional device. At $1 \mu \mathrm{m}$ overall thickness they are able of conformal adhesion to the body surface, acting as a second skin. ${ }^{[15]}$ At lower thicknesses, in the range of few hundreds nm to one micron, they show to achieve self-adhesion to arbitrary 3D surfaces with no-gap conformal contact.[16] Therefore, the way in which these tattoo electrodes are able to create a tight contact interface is strongly related to their physical features, which in turn are linked to the materials and processes adopted in their fabrication.[15] Various types of electrodes create various ways of contact with the skin primarily assessed by the electrochemical impedance spectroscopy. For traditional $\mathrm{Ag} / \mathrm{AgCl}$ electrodes, an ionic gel maintains a hydrated interface with the epidermis. In this case, the signal transduction is based on traditional electrochemical model[17] where an equivalent circuit describes the electrode-skin interface and the skin impedance. $[4,18]$ The electrode-skin interface comprises a parallel RC network. In solid dry electrodes due to the perspiration, a thin moisture layer appears at the electrode-skin interface, which replaces the ionic gel. As reported in the literature, after a few minutes from the application on skin, dry and gel electrodes exhibit the same impedance spectra. [19] For ultra-conformal conducting polymers (CPs) tattoo electrodes, the signal transduction mechanism remains unexplained. The different trend compared to the aforementioned cases is revealed from impedance spectra (in terms of shape and magnitude), and is also observable in other studies[7,20-22], albeit never rationalized. Tattoos show higher impedances over short and long-term evaluations in reference to traditional gelled electrode.[15] However, despite a five 
times higher impedance in the low frequency range, tattoo electrodes are able to record low amplitude signals related to brain activity, with an excellent signal quality, comparable with state of the art electrodes. ${ }^{[23]}$ This divergence motivated the present study to understand how this new class of ultrathin conducting polymer sensors operates.

Conducting polymers have shown important improvement in lowering the contact impedance of metallic electrodes, which in turn resulted in better potential's recordings with high signal-tonoise ratio (SNR). ${ }^{[24][25]}$ This is generally attributed to their mixed (ionic-electronic) conductivity and structural porosity.[26,27] The most used $\mathrm{CP}$ in bioelectronics is the poly(3,4ethylenedioxythiophene) polystyrene sulfonate (PEDOT:PSS). It shows a purely capacitive behavior in storing charges at the electrode in an electrolyte interface.[28] In implantable electrodes using CPs as an electroactive coating, comparisons are made in reference to the underling metal electrode applying electrochemical analysis in an electrolytic buffer solution.[29] In cutaneous sensors, such a deterministic approach is missing. Here we aim to contribute to the systematic understanding of skin-electrode interface designed for unobtrusive biomonitoring through a close contact interface. We propose a model describing how the ultrathin and conformable PEDOT:PSS tattoo electrodes transduce biological potentials from the epidermis. The recording of electrochemical impedance spectra (EIS) for standard gelled $\mathrm{Ag} / \mathrm{AgCl}$, tattoo and gel-combined tattoo electrodes advocates the transfer mechanism based on modeled equivalent electrical circuit components. Additional recordings of impedance profiles with a water layer at the electrode-skin interface, mimicking perspiration, as well as on multiple subjects allow simulating eventual interface possibilities. Through this approach, we 
exhaustively explain the implication of a complete dry interface in relation to the electrodes' long-term use, inter/intra-subject variations and the signal coherence.

\section{Results and Discussion}

In cutaneous electrophysiology, the skin impedance changes with respect to the skin hydration. [30-32] The complexity of the skin model encompasses various layers where the epidermis overlays the inner dermis, which overheads the subcutaneous tissue, composed of the hypodermis and the muscle layer (Figure 1a). The topmost layer is the stratum corneum (SC) that is primarily made of up to 20 layers of dead cells (corneocytes), sandwiched in a lipid domain.[31] Due to its composition, this layer is dry, hydrophobic, stiff and more electrically insulating than the underlying ones. Therefore, electrophysiological signals propagate within the deeper layers of the skin, such as dermis and the hypodermis.[3]

The developed models for the transduction of biosignal through the layered skin structure have two main components; one represents the skin impedance $\left(Z_{\text {skin, }}\right.$, Figure 1a) and the second describes the electrode-skin interface $\left(Z_{\text {int }}\right.$, Figure $\left.1 \mathbf{b}\right)$. In Figure $1 b$ the two equivalent circuits involved in the signal transduction through dry tattoo and $\mathrm{Ag} / \mathrm{AgCl}$ electrodes are shown consequently. In the case of tattoo electrodes, our hypothesis is that the signal passes through a real capacitor, where the dry SC layer is a dielectric component of the capacitor, with a parasitic resistor representing sweat ducts (Figure $1 \mathrm{~b}$, bottom). For $\mathrm{Ag} / \mathrm{AgCl}$ electrodes, the model considered is state of the art, with signal transduction taking place inside the gel through exchange of $\mathrm{Ag}^{+}$and $\mathrm{Cl}^{-}$ions (Figure 1b, top). The models are composed of passive (e.g resistor and capacitor) and distributed (Constant Phase Element - CPE) elements built to fit the EIS 
recordings performed on the forearm, in the typical three-electrode configuration. Schematics of three-electrode configuration (with the counter (CE), the reference (RE) and the working (WE), electrodes) is displayed in Figure 2a. This configuration measures the global impedance $\left(Z_{\text {skin }}+\right.$ $Z_{\text {int }}$ related to sole WE.[33] Figure $2 \mathbf{b}$ shows the tattoo and the $\mathrm{Ag} / \mathrm{AgCl}$ electrodes used for the impedance recordings. A close-up view of a tattoo with a printed PEDOT:PSS line shows its conformal adhesion to the skin.

The skin impedance, $Z_{\text {skin }}$ is exemplified by a traditional Cole model [34] consisting of a resistor $\left(R_{s}\right)$ in parallel with the $C P E_{s}$ and then in series with a resistor $\left(\mathrm{R}_{\infty}\right)$ representing the underlying layers (Figure 2a). We adopted a simplified model, with one parallel network, to highlight and understand the effect of the electrode-skin interface. $R_{s}$ is the electrical resistance of the skin which is strongly dependent on the skin hydration state[35]; same dependence is true for the $\mathrm{CPE}_{\mathrm{s}}$, an empirical element considered as a capacitor with an inhomogeneous surface.[36] In general, the impedance of CPE can be expressed as follows:

$Z_{C P E}=\frac{1}{Y_{0}(j w)^{\alpha}}$

where $\mathrm{Y}_{0}$ is the admittance $(\mathrm{S}), \mathrm{j}$ is the imaginary unit, $\omega$ is the angular frequency of the signal $(\omega$ $=2 \pi \mathrm{f}, \mathrm{f}$ being the frequency) and $\alpha$ is a dimensionless constant ranging between 0 and 1 . When $\alpha$ $=1$ the $\mathrm{CPE}$ is an ideal capacitor, while when $\alpha=0$ the $\mathrm{CPE}$ is a purely resistive element.

The $\mathrm{R}_{\infty}$ is the only invariant in these parametric models. We estimate its value through impedance measurements in a four-electrode configuration (Figure 2c), a method to quantify the inner skin resistivity. ${ }_{[37,38]}$ From the four-electrode recordings, a frequency-dependent behavior is noticed with a cutoff frequency at $10^{3} \mathrm{~Hz}$. The equivalent circuit is a parallel network of $\mathrm{R}_{\infty}$ and a 
$\mathrm{CPE}$ (Figure 2d). The big capacitor $\left(\mathrm{Y}_{0}(\mathrm{CPE})=5.38 \mu \mathrm{S}\right)$ results in a small effect on the overall impedance, therefore we fix the $\mathrm{R}_{\infty}$ value at $50 \Omega$ in all models.

To model the electrode-skin interface three cases with a gelled $\mathrm{Ag} / \mathrm{AgCl}$ electrode, a tattoo (dry) and gel-assisted tattoo electrodes allow underlining the difference in interfacing the skin. In the gel-assisted tattoo, and before laminating the electrodes on the skin, a gel drop forms a conductive and hydrated interface. As reported in Figure 3a, and also found in previous studies, [23] the impedance spectra ( $Z$ modulus) recorded with dry tattoo and gelled electrodes differ both in shape and in magnitude. This is especially remarkable in the low frequency range $(\mathrm{f}<10 \mathrm{~Hz})$. We link these differences to the transduction mechanism (which affects the $\mathrm{Z}$ shape) and to the nature of the interface (which influences the $\mathrm{Z}$ magnitude). The Bode and Nyquist plots with their fitted models from the EIS recordings are shown in Figure $3 \mathrm{a}$ and the values of the equivalent circuit components are presented in Figure 3b (see supplementary information Figure S1 for model-estimated errors).

The established contact of the gelled $\mathrm{Ag} / \mathrm{AgCl}$ electrode is generally described with a common model: a parallel network of a capacitor $\left(\mathrm{C}_{\mathrm{dl}}\right)$ and a resistor $\left(\mathrm{R}_{\mathrm{f}}\right)$, followed by a series resistor $\left(\mathrm{R}_{\mathrm{c}}\right.$.. ${ }^{[18]}$ The $\mathrm{C}_{\mathrm{dl}}$ is the double layer capacitor of the electrolyte gel. The $\mathrm{R}_{\mathrm{f}}$ represents the charge transfer due to the faradaic current that is established at the electrode/gel electrolyte interface (Figure $1 \mathrm{~b}$ ). Finally, the $\mathrm{R}_{\mathrm{c}}$ is the overall in-series resistance of the gel and of the external connection.

The tattoo gel-assisted interface follows the same model. The PEDOT:PSS tattoo establishes a double layer capacitance at the gel level where small faradaic currents can take place with $\mathrm{R}_{\mathrm{f}} \sim$ 
few $\mathrm{k} \Omega$. Here, we do not exploit the mixed conductivity of the $\mathrm{CP}$ electrodes, as we operate in a low voltage range $(0.1 \mathrm{~V}$ sinusoidal voltage).[26,27]

The equivalent circuit of tattoo dry interface reiterates the same basic components but with a complete different meaning. Tattoo electrodes mirror the signal as a capacitor $\left(\mathrm{C}_{\mathrm{sc}}\right)$, having the first layer of the dry SC acting as a dielectric $(800 \mathrm{~nm}$ - the thickness of one layer of corneocytes ${ }^{[39]}$ and the electrode as one of the two-capacitor plates. The other capacitor plate is located at the interface with the remaining SC and epidermis (Figure 1b). This concept is largely adopted for the measurement of SC hydration using commercial products. ${ }^{[40]}$ The $\mathrm{R}_{\mathrm{p}}$ represents the parasitic resistance due for example to sweat ducts in the skin.[35] The series resistor $\left(\mathrm{R}_{\mathrm{c}}\right)$ includes the resistance of the external connection. This rationalization is reasonable under the hypothesis of a fully dry interface, as it is the case here.

To understand the physical meaning of the models we compare impedance curves of different electrodes shown in Figure 3. The pronounced capacitive behavior of the dry tattoo electrodes is visible in both the $\mathrm{Z}$ modulus and the Nyquist plot in Figure 3a. However, the dry tattoo electrodes do not show the typical semi-circle trend, ${ }^{41]}$ as in the case of $\mathrm{Ag} / \mathrm{AgCl}$ and tattoo gel electrodes, revealing their large capacitance. The $\mathrm{C}_{\mathrm{sc}}$ and the $\mathrm{CPE}_{\mathrm{s}}$ were actually found around 1 - 1.5 orders of magnitude lower than the corresponding elements calculated for earlier mentioned two wet electrodes. Moreover, the resistive elements in dry tattoo show a larger value (around 2 orders of magnitude) explaining the large $\mathrm{Z}$ modulus at quasi-dc regime (1-10 Hz) also observed in previous results. ${ }^{[15,23]}$ Indeed, at low frequencies, the capacitors tend to an open circuit behavior and the resistive elements became dominant in the model. 
These differences are rationalized from two points of view, one regarding the skin model and another regarding the electrode-skin interface. From the skin model perspective, the hydrated SC present with the wet electrodes leads to a lower $R_{s}$ value (Figure $3 b$ ), a result of the higher ion mobility at the interface. The $R_{s}$ reached around $1 \mathrm{M} \Omega \mathrm{cm}^{-2}$ in a gelled electrode and $17{\mathrm{M} \Omega \mathrm{cm}^{-2}}^{-2}$ in the dry tattoo. These values are in agreement with the trend reported in the literature, where higher $R_{s}$ is observed for dry electrodes (with respect to wet interface) placed on the forearm. [35] Regarding the $\mathrm{CPE}_{\mathrm{s}}$ in Figure $3 \mathrm{~b}, \alpha$ is around 0.8 in all models, consistent with skin impedance studies. ${ }^{[31,34]}$ From the $\mathrm{CPE}_{\mathrm{s}}$ it is possible to extract the pseudo-capacitance values: $6.1 \mathrm{nF} \mathrm{cm}^{-2}$ for dry tattoo, $73 \mathrm{nF} \mathrm{cm}{ }^{-2}$ for gel-assisted tattoo and $174 \mathrm{nF} \mathrm{cm}^{-2}$ for $\mathrm{Ag} / \mathrm{AgCl}$ electrode (see supplementary information Figure S2 for pseudo-capacitance calculation). The values are in the range of previously reported data $\left(20-60 \mathrm{nF} \mathrm{cm}^{-2}\right)$ and consistent with other studies that clearly indicated an increase of SC capacitance due to the increased water content in the skin. ${ }^{[31,42,43]}$ Moreover, in wet interfaces the gel sprawl on the skin leads to an enhanced effective contact area that additionally increase the skin capacitance value. Finally, the admittance of the $\mathrm{Ag} / \mathrm{AgCl}$ electrode's model $\left(\mathrm{Y}_{0}=2.310^{-7} \mathrm{~S} \mathrm{~cm}^{-2}\right)$ is comparable with previous results ${ }^{[44]}$ confirming the consistency of the used model.

From the electrode-skin interface perspective, the $\mathrm{C}_{\mathrm{sc}}$ value $(18.9 \mathrm{nF} \mathrm{cm}-2)$ is comparable with the theoretical value $(11 \mathrm{nF} \mathrm{cm}-2$, Figure $3 b)$ calculated considering the dielectric constant $\left(\varepsilon_{\mathrm{r}}\right)$ equal to 10 [31,45], the area of $1 \mathrm{~cm}^{2}$ and the dielectric thickness equal to $800 \mathrm{~nm}$ (the thickness of one corneocytes layer). Thereafter, a good agreement with the theoretical calculation validates our proposed model. The $\mathrm{C}_{\mathrm{sc}}$ value was found seven times higher in the gel-assisted tattoo model $\left(142 \mathrm{nF} \mathrm{cm}^{-2}\right)$. This increment takes account of two considerations. First, the $\varepsilon_{\mathrm{r}}$ value increases 
by a factor of five when passing from a dry to a wet interface (being around 50 for wet SC [46]). Second, the effective area involved in the capacitor estimation is bigger due to the gel spreading at the skin interface. ${ }^{[47]}$ The derived $\mathrm{C}_{\mathrm{dl}}$ value for the $\mathrm{Ag} / \mathrm{AgCl}$ electrode $(238 \mathrm{nF} \mathrm{cm}-2)$ is in agreement with other results. ${ }^{[44]}$ Looking at the $\mathrm{Z}$ modulus (Figure 3a), the dry tattoo exhibits higher values in quasi-DC condition, because of the higher resistive elements, and it did not show the typical plateau visible in gelled electrodes. In wet interfaces there is a pole (at cutoff frequency of the $\mathrm{RC}$ circuit, $\mathrm{f}_{\mathrm{co}}=1 / 2 \pi \mathrm{RC}=17 \mathrm{~Hz}$ ) in the transfer function shifted with respect to the dry tattoo $\left(a t f_{c o}=0.11 \mathrm{~Hz}\right.$ ) due to the different values in the electrode-skin interface circuit. Looking at high frequencies ( $\mathrm{f}>10^{4} \mathrm{~Hz}$ ) both gelled and dry tattoos tend to the same value (Figure 3a, $\mathrm{Z}$ modulus), which is slightly bigger than the one reached by the $\mathrm{Ag} / \mathrm{AgCl}$ electrode. At these frequencies, all the capacitors behave like a short circuit, thereafter the main contribution to the $Z$ modulus is in the series resistors, $R_{c}$ and $R_{\infty}$. As $R_{\infty}$ is fixed at $50 \Omega$, the $R_{c}$ is the main accountable element of the high frequency resistance. Tattoo electrodes exhibits the highest value, $R_{c}=883 \Omega \mathrm{cm}^{-2}$. This takes into account the external contact, here provided through a mechanical coupling of a PEDOT:PSS thin layer at the clip connector, shown in Figure $\mathbf{S 3} \mathbf{b}$ and $\mathbf{c}$. In the case of $\mathrm{Ag} / \mathrm{AgCl}$ electrodes, the contact takes place through a fitted metallic connector, being more conductive than the polymeric one.

From the phase and Nyquist plots (Figure 3a), it is possible to notice a modest deviation of the model with respect to the data. The error curves in Figure S1 additionally support this observation. The model adopted to describe the skin is composed of one generalized parallel network $\left(\mathrm{R}_{\mathrm{s}}-\mathrm{CPE}_{\mathrm{s}}\right)$ explaining possibilities of such deviations. Skin exemplification is challenging owing to the inhomogeneous nature of human tissues. ${ }^{[35]}$ Here we decided to use a 
basic representation that allows a straightforward comparison between the three models and their physical data. Nonetheless, relevant errors are in the range of $10 \%$.

In Figure 4 we present a proof of a complete dry and stable interface achieved with tattoo electrodes. The perspiration is a common process of the body functioning and can critically affect the performance of body-worn devices. In skin adhesive electrodes, sweat accumulation can lead to their delamination and to relative displacement, with obvious opposing effects in the quality of surface recordings. In order to understand how tattoos respond in daily use, we record EIS with dry tattoo and $\mathrm{Ag} / \mathrm{AgCl}$ electrodes over two hours. To mimic the electrochemical response of the tattoo electrodes with perspiration we record impedances on the wet skin, where a drop of water is deposited on the skin just before the electrode release.

In Figure 4a we show the recordings, right after the application of the electrodes ( 0 hours) and 2 hours later. After 2 hours, we obtain identical plots over the whole frequency spectrum. The presence of a sweat layer during these recordings would have changed the shape of the curves with time reaching the behavior of the gelled electrodes (Figure 3a). The addition of water at the interface did not result in $\mathrm{Z}$ modulus or phase modification, as shown in Figure $\mathbf{4 b}$. These observations indicate that PEDOT:PSS tattoo electrodes can uptake the water or perspiration by its thin porous structure. We base this assumption on the recently reported work claiming that tattoo electrodes create a breathable interface with the skin. ${ }^{[1,7,10]}$ As water vapor transmission rate (WVTR) increases with decreasing thickness, relatively thick and impermeable substrates represent a physical barrier for fluids/vapours at the skin interface. This can cause skin irritation, loss of long-term functioning and impaired sensors operation. Therefore, ultrathin tattoo 
electrodes are able to sustain stable, reliable and long lasting applications as permeable selfadhesive surfaces.

Inter-subject and intra-subject impedance recordings allow the evaluation of the physiological and environmental fluctuations interfering with the signal recordings with respect to dry and wet interfaces. The impedance varies in response to physiological changes, as body hydration, and/or to peripheral conditions, as humidity and temperature of the surrounding environment. ${ }^{[15,48]}$ The impact of these parameters are critical to control, especially in daily-use conditions (i.e. outside of controlled hospital room environment). EIS recordings with dry tattoo and $\mathrm{Ag} / \mathrm{AgCl}$ electrodes on three different subjects and on the same subject with different electrodes (two diverse tattoos and $\mathrm{Ag} / \mathrm{AgCl}$ electrodes), at different epochs (i.e. in two days) shown in Figure $4 \mathbf{c}$ and $\mathbf{d}$. The results indicate a minor variation in tattoo electrodes' impedance, in comparison with $\mathrm{Ag} / \mathrm{AgCl}$ electrodes (Figure $4 \mathrm{c}$ and $\mathrm{d}$ with the $\mathrm{Z}$ modulus at $1 \mathrm{~Hz}$ listed in Table 1). This study demonstrates that a complete dry tattoo interface is less sensitive to an eventual body perspiration and to environmental variations, therefore ensuring a stable signal transmission. This reduces the issues related to the electronics read-out design, especially in view of wearable devices development.

Table 1. Values of the impedance moduli at $1 \mathrm{~Hz}(\Omega)$, extracted from recordings performed with $\mathrm{Ag} / \mathrm{AgCl}$ and tattoo electrodes on the same subject in diverse set-ups (diverse electrodes and days) and on different subjects.

Figure 1. The skin and the electrode interface. a) Schematization of the skin layers. The 
epidermis, with the stratum corneum as top layer and the electrodes adopted in the study: tattoo and $\mathrm{Ag} / \mathrm{AgCl}$ electrodes. The dermis, with sweat glands, nerve ending and blood vessels. The subcutaneous tissue, composed by the hypodermis and the muscle layer. On the top-right, the equivalent circuit is adopted to model the skin. b) the electrode/skin interface through $\mathrm{Ag} / \mathrm{AgCl}$ (top) and tattoo electrode (down). The equivalent circuits are represented together with the physical mechanism leading to the biosignal transduction.

Figure 2. The experimental set-up. a) Schematization of the three-electrode configuration adopted in impedance recordings, and the equivalent circuit of the skin. Recordings performed in potentiostatic mode, a small $(0.1 \mathrm{~V}$ amplitude) sinusoidal signal $(0.1-105 \mathrm{~Hz})$ is supplied through the CE-WE couple. As current flows between the CE-WE couple, the potential variation is measured across the RE-S couple. b) The electrodes worn on the skin. Left: tattoo and $\mathrm{Ag} / \mathrm{AgCl}$ electrodes adopted in the impedance recordings. Right: a tattoo released on the finger, evidencing ultraconformable adhesion. Scale bar $3 \mathrm{~mm}$. c) Schematization of the four-electrode configuration adopted in the $\mathrm{R}_{\infty}$ estimation. Same recording set-up as described in a). d) Recorded impedance data (light pink) and model (dark pink) of $\mathrm{R}_{\infty}$, in four-electrode configuration, with modulus (left axis) and phase (right axis) representation.

Figure 3. Modelling of the skin-electrode interface. a) Modulus, phase and Nyquist plots $(\omega=$ $2 \pi \mathrm{f}$ ) of the impedance data and the developed model for the three analyzed electrodes (Tattoo dry, Tattoo gel and $\mathrm{Ag} / \mathrm{AgCl}$ electrode). b) The three models built to fit the impedance data recordings for the three electrodes analyzed, with the extrapolated value of the electronic component (value calculated for $1 \mathrm{~cm}^{2}$ area). 
Figure 4. Impedance variation over time and over subjects. a) Impedance modulus over the recorded frequency range and its variation with time; the data recorded with tattoo electrodes and $\mathrm{Ag} / \mathrm{AgCl}$ electrodes at $\mathrm{Oh}$ and $2 \mathrm{~h}$ since placement on skin. b) Impedance modulus and phase of tattoo electrodes (tattoo dry) and tattoo electrodes placed over wet skin (tattoo water). c,d) Impedance modulus (c) and phase (d) recorded with tattoo and $\mathrm{Ag} / \mathrm{AgCl}$ electrodes on three different subjects $(1,2,3)$ and on the same subject at different times: day 1, day 2 .

\section{Conclusion}

In summary, we model the signal transduction mechanisms in ultraconformable conducting polymer tattoo electrodes. By finding a good agreement between the fitted and the acquired impedance data, we reveal that dry tattoo electrodes pair with biological signals through a capacitive interface in which the upper SC layer acts as the dielectric. This is different from what occurs in standard $\mathrm{Ag} / \mathrm{AgCl}$ electrodes, where the signal transduction takes place in its gel layer. The capacitive coupling explains why the high values of $\mathrm{Z}$ modulus, found at mid-low frequencies $\left(<10^{2} \mathrm{~Hz}\right.$, the frequencies of interest in surface electrophysiology), are not a limiting factor in the intrinsic ability of the conducting polymer electrode to transfer the biosignals. Based on the stability and inter subject's evaluations we observe that tattoo electrodes establish a completely dry and stable contact. The benefit of this configuration allow suppressing capacitance changes at the interface in a movement. As result, it enables to reach good and artifacts free recordings, which are impossible with macroscopic and gelled state of the art electrodes. This study provides a quantitative description of the skin vs. dry conformal electrode interface. It contributes to the large development of ultrathin tattoo-based or tattoo-like sensors in 
the field of conformable electronics, where the imperceptible and long-lasting devices are of high interest for clinical relevance in personalized medicine. 


\section{Experimental Section}

4.1 Materials. PEDOT:PSS aqueous dispersion (Clevios ${ }^{\mathrm{TM}}$ PJet 700 by Heraeus), was used as conductive ink. Commercially available temporary tattoo paper kit (Silhouette America, Inc, US) was adopted as substrate (see supporting information Figure S4 for details). Polyethylene naphthalate (PEN) thin-film (Goodfellow, $1.3 \mu \mathrm{m}$ thickness) was employed as flat interconnection and Polyimide (PI) tape (Kapton ${ }^{\circledR}$ tape by $3 \mathrm{M}, 50 \mu \mathrm{m}$ thickness) as back support for the external connection. The 3D printing of the clip-connector is made of Acrylonitrile Butadiene Styrene (ABS).

4.2 Tattoo Electrodes fabrication. The process here adopted follows the previously reported strategy ([15]). Briefly, tattoo electrodes are fabricated by inkjet printing (Dimatix DMP-2800 system; Fujifilm Corp., Japan) of PEDOT:PSS ink onto tattoo paper and PEN film to manufacture respectively the electrodes and the interconnections. Thereafter a lamination process enables the final assembly. $\mathrm{A} \mathrm{CO}_{2}$ laser cutter (VLS 3.50, $50 \mathrm{~W}$; Universal Laser Systems) is used for the shaping of each component with optimized power, speed, resolution and focus distance for each different material in order to provide a sharp and neat cut. Figure S3a details the tattoo electrodes geometry and layout.

4.3 Impedance recordings and model development. The model elaboration is based on impedance recordings, performed with an Autolab potentiostat (Metrohm Autolab B.V.) and its associated software NOVA version 2.1.2. The measurements are executed in three electrodes configuration, with $2.5 \mathrm{~cm}$ interspaced center-to-center electrodes. The counter (CE) and the Reference (RE) electrodes were always $\mathrm{Ag} / \mathrm{AgCl}$ electrodes (Ambu BlueSensor, REF M-00-S/ 50), while the Sensing (S) and the Working Electrode (WE) were short-circuited and connected 
to the electrode of interest, tattoo or $\mathrm{Ag} / \mathrm{AgCl}$ electrode (see supporting information, Figure S5). All the impedance measurements are performed on the forearm of a volunteer. All the electrodes had an area of $1 \mathrm{~cm}^{2}$. The measurements are done in potentiometric mode with an applied sinusoidal signal of $1 \mathrm{mV}$ amplitude. The frequency range was set between 0.1 and $1 \mathrm{MHz}$. All the recordings are performed few minutes after the electrodes application. This the time is needed for tattoo electrodes to reach a steady state following their transfer on skin using waterassisted delamination process.

The data reported in Figure 2 and 3 are representative recordings of singular electrodes, showing the typical behaviour observed in multiple acquisitions ( $\sim 13$ recordings for dry tattoo and $\mathrm{Ag}$ / $\mathrm{AgCl} ; 5$ recordings for gel tattoo; 3 recordings for tattoo water and 4 recordings for the $2 \mathrm{~h}$ duration), data not shown. The data in Figure 4C are from 3 different electrodes for type (Tattoo $1,2,3 ; \mathrm{Ag} / \mathrm{AgCl} 1,2,3)$ on different subjects.

4.4 Experiments involving human subjects. This research does not include the collection of identifiable private information related to the individual's health status and concerns only the technological demonstration. Three able-bodied subjects ( 1 males and 2 females aged $26.7 \pm 5.9$ years old) free of any motor disorders participated in this study. Informed consent in accordance with the Declaration of Helsinki was obtained before conducting the experiments from each subject. One of the participants performed the impedance recording for the model development. The same participant, together with other two subjects, volunteered in the impedance evaluations for inter-subject impedance variation. 



\section{Acknowledgments}

This work was supported by the BPIFrance AUTONOTEX project, and an ANR JCJC OrgTex project ANR-17-CE19-0010, E.I. wishes to thank CMP cleanroom staff for their technical support during the development of the project as well as MicroPacks platform at the Centre Microelectronics in Provence.

\section{Conflict of Interest}

The authors declare no conflict of interest.

\section{Supporting Information}

In the supporting information we describe the model errors (S1), the pseudo capacitance calculation (S2), the tattoo electrodes' fabrication, a layout including the external connection (S3), the adopted Temporary Tattoo Kit (S4) and illustrate the experimental set-up for impedance evaluations (S5) .

\section{References}

[1] L. M. Ferrari, K. Keller, B. Burtscher, F. Greco, Multifunctional Materials 2020, 3, 032003.

[2] R. A. Nawrocki, H. Jin, S. Lee, T. Yokota, M. Sekino, T. Someya, Advanced Functional Materials 2018, 28, 1803279.

[3] J. Feher, Quantitative Human Physiology: An Introduction, Academic Press, 2012.

[4] Y. M. Chi, T.-P. Jung, G. Cauwenberghs, IEEE Rev. Biomed. Eng. 2010, 3, 106.

[5] S. Tian, W. Yang, J. M. Le Grange, P. Wang, W. Huang, Z. Ye, Global Health Journal 2019, 
3,62 .

[6] P. Cosseddu, M. Caironi, Organic Flexible Electronics: Fundamentals, Devices, and Applications, Woodhead Publishing, 2020.

[7] L. Tian, B. Zimmerman, A. Akhtar, K. J. Yu, M. Moore, J. Wu, R. J. Larsen, J. W. Lee, J. Li, Y. Liu, B. Metzger, S. Qu, X. Guo, K. E. Mathewson, J. A. Fan, J. Cornman, M. Fatina, Z. Xie, Y. Ma, J. Zhang, Y. Zhang, F. Dolcos, M. Fabiani, G. Gratton, T. Bretl, L. J. Hargrove, P. V. Braun, Y. Huang, J. A. Rogers, Nat Biomed Eng 2019, 3, 194.

[8] F. Stauffer, M. Thielen, C. Sauter, S. Chardonnens, S. Bachmann, K. Tybrandt, C. Peters, C. Hierold, J. Vörös, Adv. Healthc. Mater. 2018, 7, e1700994.

[9] S. M. Lee, H. J. Byeon, J. H. Lee, D. H. Baek, K. H. Lee, J. S. Hong, S.-H. Lee, Sci. Rep. 2014, 4, 6074.

[10] A. Miyamoto, S. Lee, N. F. Cooray, S. Lee, M. Mori, N. Matsuhisa, H. Jin, L. Yoda, T. Yokota, A. Itoh, M. Sekino, H. Kawasaki, T. Ebihara, M. Amagai, T. Someya, Nat. Nanotechnol. 2017, 12, 907.

[11] K. D. Guzman, K. De Guzman, G. Al-Kharusi, T. Levingstone, A. Morrin, Analytical Methods 2019, 11, 1460.

[12] A. Zucca, C. Cipriani, Sudha, S. Tarantino, D. Ricci, V. Mattoli, F. Greco, Adv. Healthc. Mater. 2015, 4, 983.

[13] L. Bareket, L. Inzelberg, D. Rand, M. David-Pur, D. Rabinovich, B. Brandes, Y. Hanein, Sci. Rep. 2017, 7, 41345.

[14] S. Kabiri Ameri, R. Ho, H. Jang, L. Tao, Y. Wang, L. Wang, D. M. Schnyer, D. Akinwande, N. Lu, ACS Nano 2017, 11, 7634. 
[15] L. M. Ferrari, S. Sudha, S. Tarantino, R. Esposti, F. Bolzoni, P. Cavallari, C. Cipriani, V. Mattoli, F. Greco, Adv. Sci. 2018, 5, 1700771.

[16] R. A. Nawrocki, Advanced Functional Materials 2019, 29, 1906908.

[17] A. J. Bard, Bard, L. R. Faulkner, Electrochemical Methods: Fundamentals and Applications, John Wiley \& Sons Incorporated, 1980.

[18] M. Neuman, Electrical Engineering Handbook 1999, DOI 10.1201/9781420049510.sec5.

[19] A. Searle, L. Kirkup, Physiol. Meas. 2000, 21, 271.

[20] R. Matsukawa, A. Miyamoto, T. Yokota, T. Someya, Adv. Healthc. Mater. 2020, 9, e2001322.

[21] P. A. Lopes, D. Vaz Gomes, D. Green Marques, P. Faia, J. Góis, T. F. Patrício, J. Coelho, A. Serra, A. T. de Almeida, C. Majidi, M. Tavakoli, Adv. Healthc. Mater. 2019, 8, e1900234.

[22] A. A. Chlaihawi, B. B. Narakathu, S. Emamian, B. J. Bazuin, M. Z. Atashbar, Sensing and Bio-Sensing Research 2018, 20, 9.

[23] L. M. Ferrari, U. Ismailov, J.-M. Badier, F. Greco, E. Ismailova, npj Flexible Electronics 2020, 4, DOI 10.1038/s41528-020-0067-z.

[24] X. Cui, J. F. Hetke, J. A. Wiler, D. J. Anderson, D. C. Martin, Sensors and Actuators A: Physical 2001, 93, 8 .

[25] M. Asplund, T. Nyberg, O. Inganäs, Polymer Chemistry 2010, 1, 1374.

[26] J. Rivnay, R. M. Owens, G. G. Malliaras, Chemistry of Materials 2014, 26, 679.

[27] D. T. Simon, E. O. Gabrielsson, K. Tybrandt, M. Berggren, Chem. Rev. 2016, 116, 13009.

[28] M. Berggren, G. G. Malliaras, Science 2019, 364, 233.

[29] V. Sankar, E. Patrick, R. Dieme, J. C. Sanchez, A. Prasad, T. Nishida, Front. Neuroeng. 
2014, 7, 13.

[30] T. Yamamoto, Y. Yamamoto, Medical \& Biological Engineering 1976, 14, 151.

[31] S. Björklund, T. Ruzgas, A. Nowacka, I. Dahi, D. Topgaard, E. Sparr, J. Engblom, Biophys. J. 2013, 104, 2639.

[32] R. T. Tregear, Physical Functions of Skin, 1966.

[33] L. A. Geddes, Ann. Biomed. Eng. 1997, 25, 1.

[34] Y. N. Kalia, R. H. Guy, Pharm. Res. 1995, 12, 1605.

[35] J. Patrick Reilly, Applied Bioelectricity: From Electrical Stimulation to Electropathology, Springer Science \& Business Media, 2012.

[36] Z. Lukács, Journal of Electroanalytical Chemistry 1997, 432, 79.

[37] S. Ha, C. Kim, Y. M. Chi, A. Akinin, C. Maier, A. Ueno, G. Cauwenberghs, IEEE Trans. Biomed. Eng. 2014, 61, 1522.

[38] S. Grimnes, Medical \& Biological Engineering \& Computing 1983, 21, 739.

[39] M. E. Johnson, D. Blankschtein, R. Langer, J. Pharm. Sci. 1997, 86, 1162.

[40] E. Alanen, J. Nuutinen, K. Nicklén, T. Lahtinen, J. Mönkkönen, Skin Res. Technol. 2004, 10, 32.

[41] J. Serup, G. B. E. Jemec, G. L. Grove, Handbook of Non-Invasive Methods and the Skin, Second Edition, CRC Press, 2006.

[42] R. O. Potts, D. B. Guzek, R. R. Harris, J. E. McKie, Arch. Dermatol. Res. 1985, $277,489$.

[43] P. Agache, S. Mary, P. Muret, A. M. Matta, P. Humbert, Dermatology 2001, 202, 308.

[44] Y.-H. Chen, Polymer-Based Dry Electrodes for Biopotential Measurements, ARENBERG DOCTORAL SCHOOL Faculty of Engineering Science, 2016. 
[45] T. Rosendal, Acta Physiologica Scandinavica 1945, 9, 39.

[46] B. Hirschorn, M. E. Orazem, B. Tribollet, V. Vivier, I. Frateur, M. Musiani, Electrochimica Acta 2010, 55, 6218 .

[47] G.-L. Li, J.-T. Wu, Y.-H. Xia, Q.-G. He, H.-G. Jin, J. Neural Eng. 2020, 17, 051004.

[48] L. Guo, M. Ma, N. Zhang, R. Langer, D. G. Anderson, Adv. Mater. 2014, 26, 1427. 
Table 1.

\begin{tabular}{|c|c|c|}
\hline @ $1 \mathrm{~Hz},[\Omega]$ & $\mathrm{Ag} / \mathrm{AgCl}$ & TTE \\
\hline $\begin{array}{l}\text { subject 1a } \\
\text { (set-up 1) }\end{array}$ & $3.0\left(10^{5}\right)$ & $6.5\left(10^{6}\right)$ \\
\hline $\begin{array}{l}\text { subject } 1 \mathrm{~b} \\
\text { (set-up 2) }\end{array}$ & $1.0\left(10^{6}\right)$ & $3.0\left(10^{6}\right)$ \\
\hline subject 2 & $4.0\left(10^{4}\right)$ & $3.0\left(10^{6}\right)$ \\
\hline subject 3 & $2.6\left(10^{5}\right)$ & $6.5\left(10^{6}\right)$ \\
\hline
\end{tabular}


Figure 1

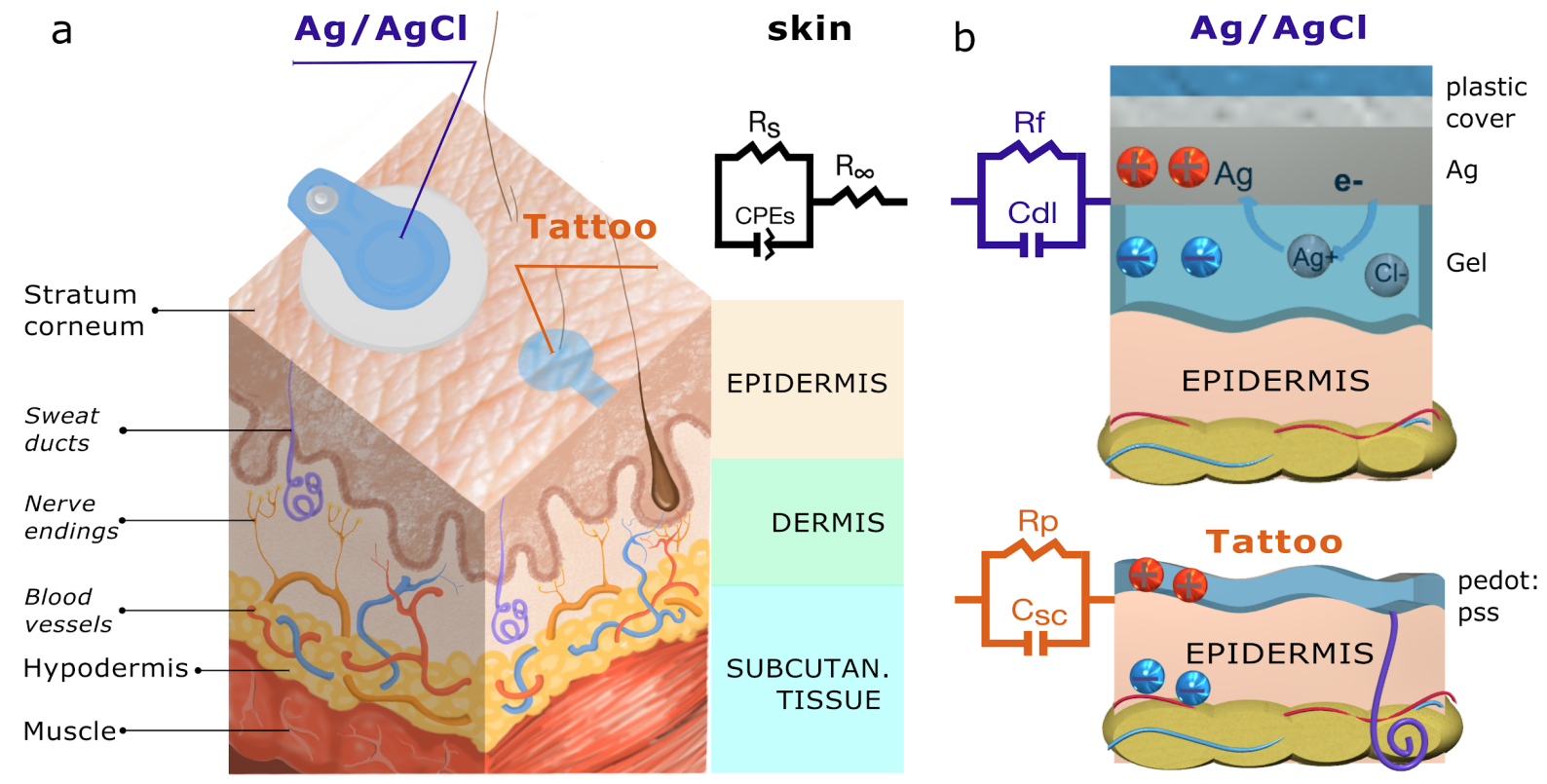

Figure 2

a

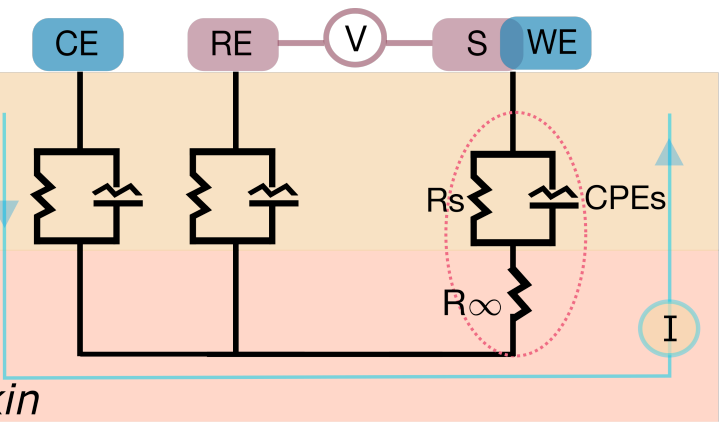

C

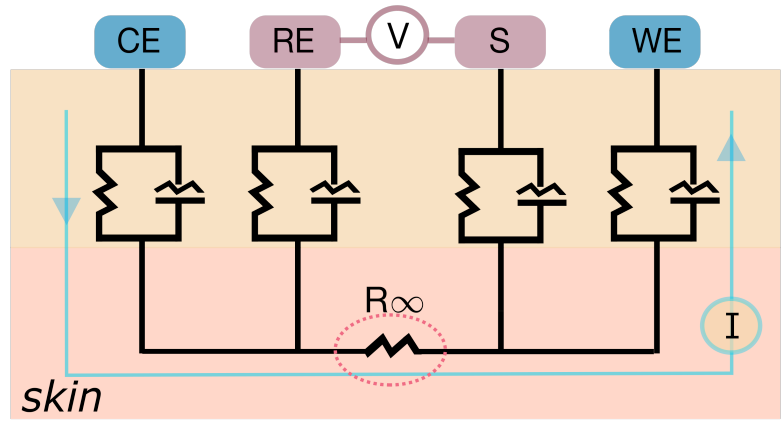

b
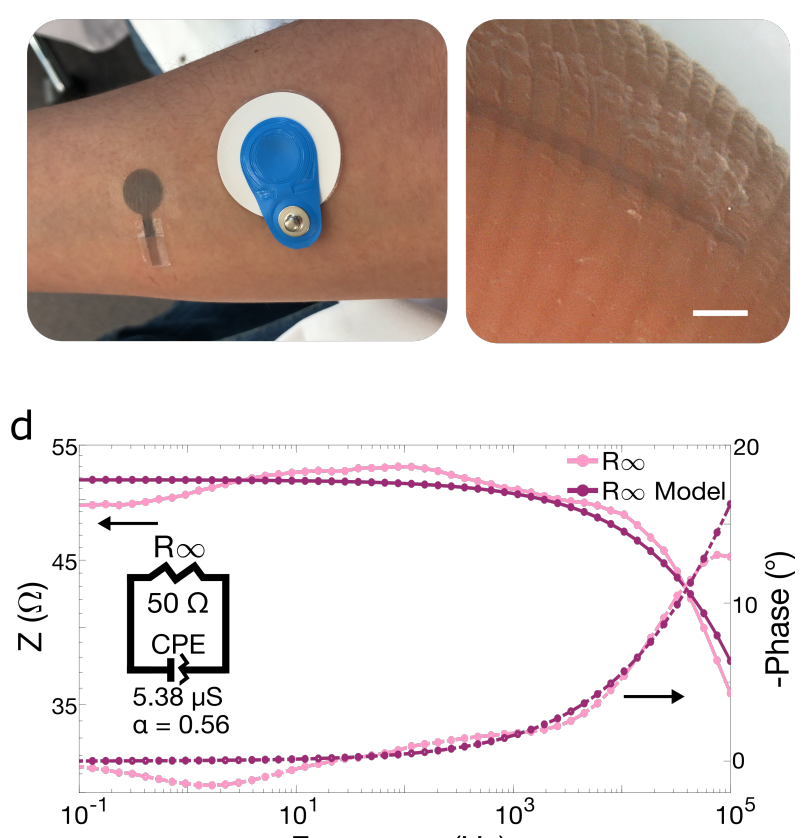

Frequency $(\mathrm{Hz})$ 
Figure 3
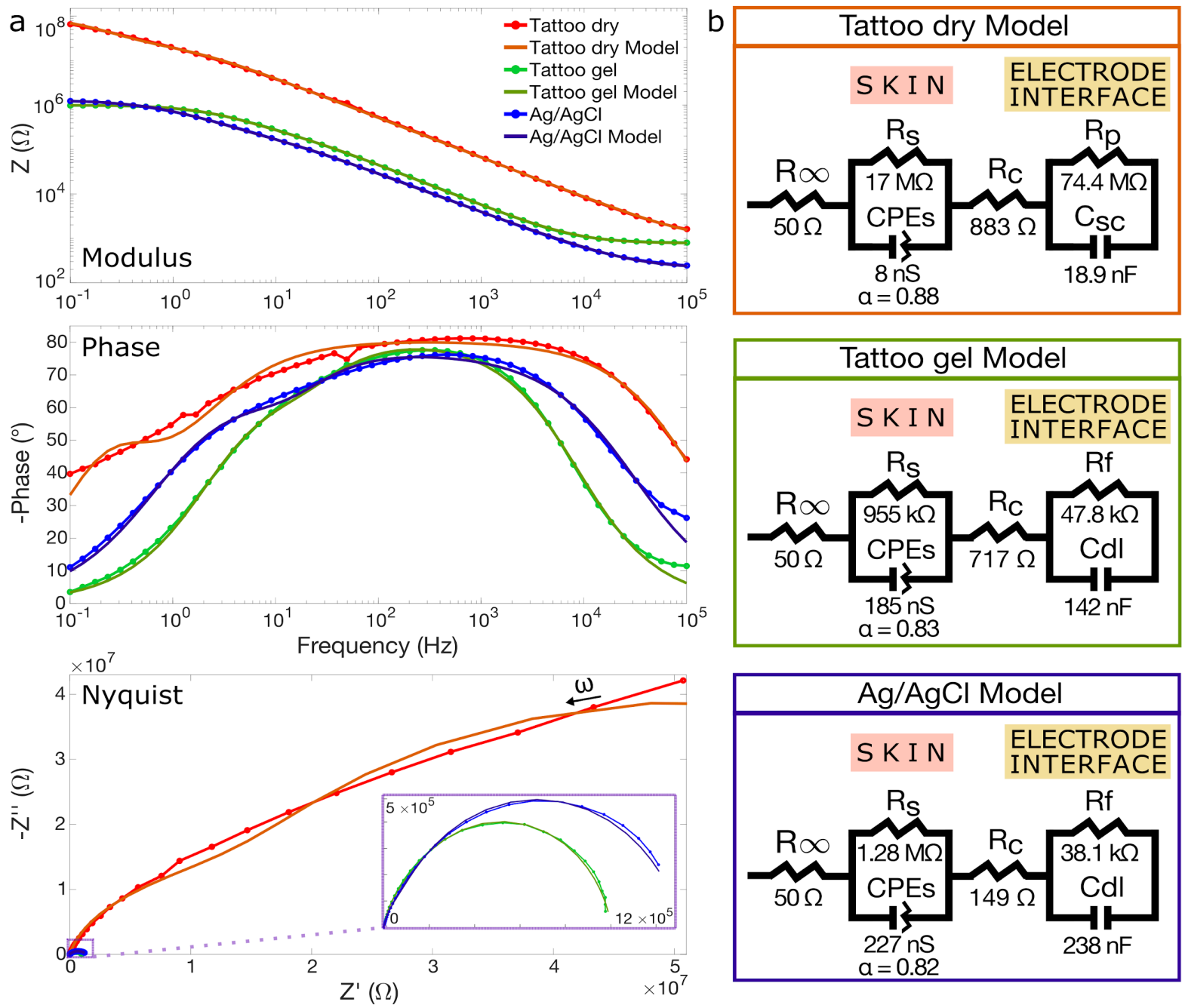


\section{Figure 4}
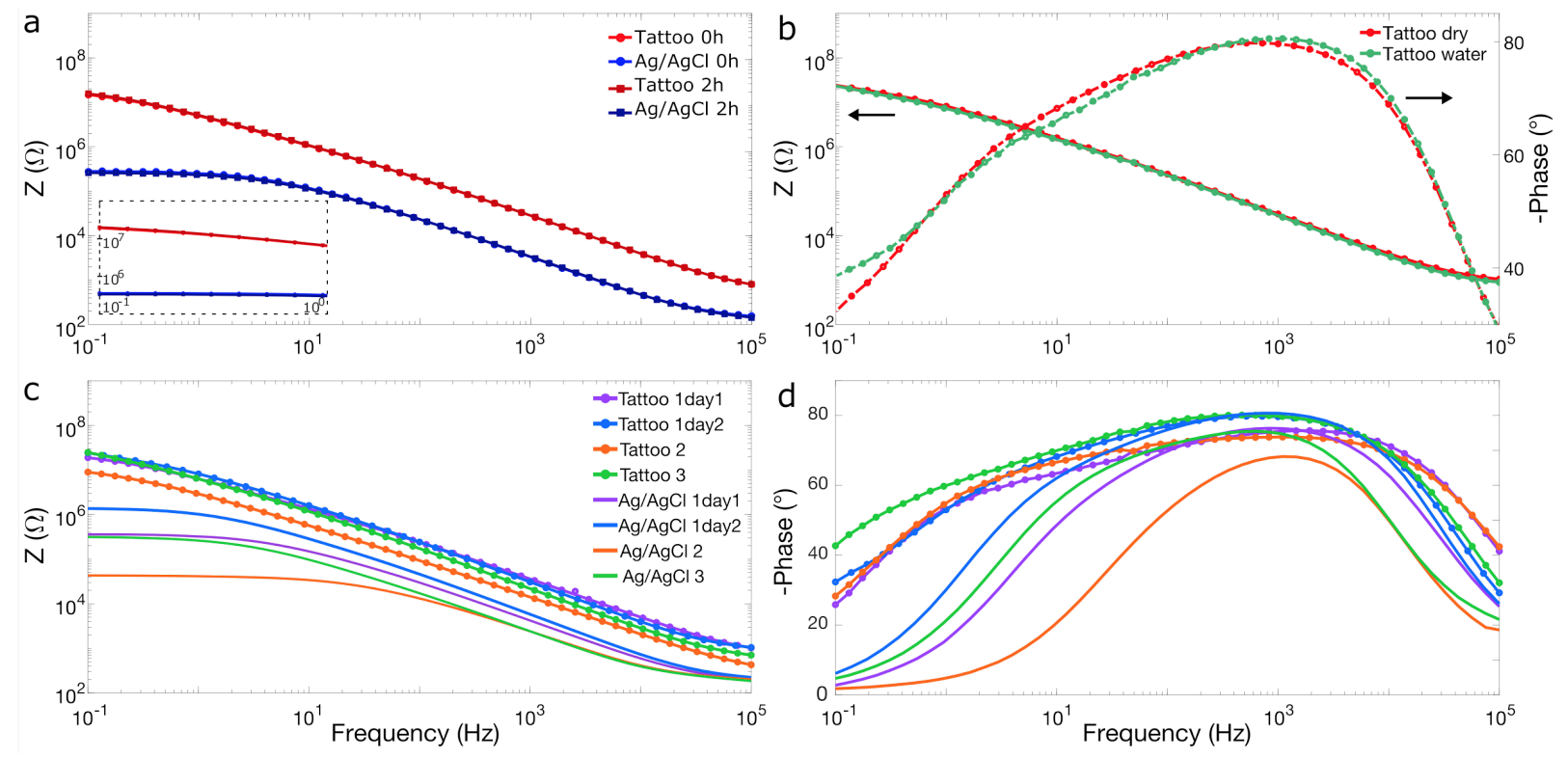


\section{Capacitive coupling of conducting polymer tattoo electrodes with the skin}

Laura M. Ferrari, Usein Ismailov, Francesco Greco and Esma Ismailova*

E-mail: ismailova@emse.fr

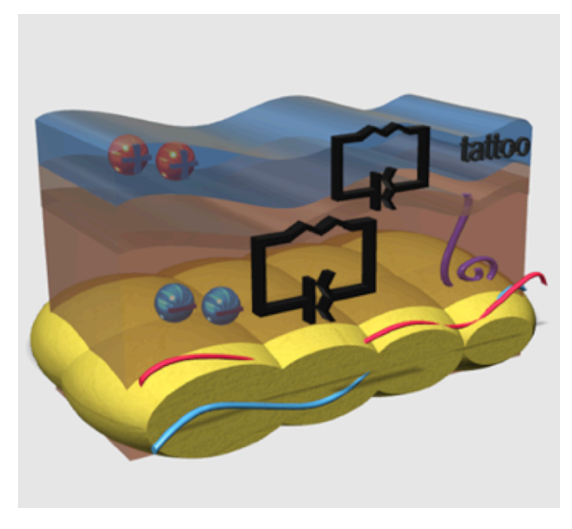

Tattoo electrodes are dry sensors that provide outstanding conformability to the skin thanks to their ultralow thickness. These electrodes interact with the skin via capacitive coupling where the upper layers act as a dielectric. Tattoos made of conducting polymer PEDOT:PSS display great mechanical stability and a long-term performance in detecting biopotentials from the skin despite their considerably large impedance. 